Courrier du Centre international Blaise Pascal

$7 \mid 1985$

Varia

\title{
Album de famille
}

Thérèse Goyet

\section{OpenEdition}

Journals

Édition électronique

URL : http://journals.openedition.org/ccibp/434

DOI : $10.4000 /$ ccibp. 434

ISSN : 2493-7460

\section{Éditeur}

Centre international Blaise Pascal

\section{Édition imprimée}

Date de publication : 5 avril 1985

Pagination : 1-12

ISSN : 0249-6674

\section{Référence électronique}

Thérèse Goyet, "Album de famille », Courrier du Centre international Blaise Pascal [En ligne], 7 | 1985, mis en ligne le 27 novembre 2015, consulté le 21 avril 2019. URL : http://journals.openedition.org/ ccibp/434; DOI : 10.4000/ccibp.434

Ce document a été généré automatiquement le 21 avril 2019

Centre international Blaise Pascal 


\title{
Album de famille
}

\author{
Thérèse Goyet
}

\section{Sur une actualité en retard, considérations}

1 « Notre reconquête historique et spirituelle se fait lentement.» (Courrier $d u$ CIBP, $\mathrm{n}^{\circ}$ 6, p. 3). De cette lenteur nos «Amis et Correspondants» auraient eu raison de s'inquiéter. Cependant ils ont noté que si le Courrier paraît en décalage par rapport au millésime qu'il affiche, ce décalage est régulier et respecte l'annualité promise. Et comme nous donnons avec soin les dates de nos événements et l'achevé d'imprimer, la conscience est en paix avec la chronologie. Mais avec ce numéro 7 le décalage dépasse ce qui nous semble admissible et nous devons des explications.

2 Oui, la mise en lumière de patrimoine pascalien progresse. Et c'est précisément parce qu'il s'opère des trouvailles que les retards se sont accumulés.

3 Venons-en donc vite à présenter nos images pour notre album.

4 Ce qui vient à nous, c'est de l'ancien, du temps même de Pascal, des créations du temps intermédiaire, et du nouveau. Et dans tous les domaines le point de départ est toujours fortuit. Quelqu'un nous a signalé... Alors il faut découvrir là où il se trouve, l'objet intéressant, obtenir une reproduction fidèle et susceptible de passer à l'imprimerie. Et puis l'enquête commence.

5 Pour les pièces nouvelles on a le bonheur de pouvoir interroger l'auteur : ses intentions personnelles nous touchent en tant que telles car elles peuvent servir d'incitation, voire d'initiation à notre propre manière de sentir. C'est un portrait daté 1963 que nous donnons aujourd'hui à regarder (fig. 1). Il est signé de Jean Guitton, de l'Académie française : référence peu banale en peinture.

6 Quant aux effigies du temps intermédiaire que nous demandons la permission d'appeler "la basse époque pascalienne», elles sont extrêmement nombreuses. Un chercheur patient, heureux collectionneur lui-même, et auvergnat, Albert Ojardias, s'en est amusé :

Aux curieux des variations, ou plutôt des déformations d'une même physionomie humaine, je conseille expressément la collection Pascal au Cabinet des Estampes [de la Bibliothèque nationale]. On y verra Pascal byronien drapé comme Ossian, inspiré 
comme Lamartine, chauve et presque aussi satanique que Baudelaire; Pascal romanesque [...]; Pascal phtisique et qui doit s'éteindre à l'automne. Mais je recommande principalement un Pascal « ami des lumières ${ }^{1}$ »

7 Cependant comme ces pièces de basse époque peuvent procéder de traces originales et qu'elles ont plus ou moins directement éduqué notre sentiment historique, nous devons à leur égard tenir compte de raisons complexes. Les anachronismes de l'imaginaire historicisant sont eux-mêmes des données de l'histoire, mais est-ce à nous d'en traiter? Quand donc ces inventions typiques se trouvent dans des collections bien connues, nous laissons à d'autres le soin de les intégrer dans la trame de la mémoire pascalienne, comme d'en faire la critique au point de vue de l'art. Mais si nous en découvrons de négligées, voire oubliées, nous croyons bon de les ramener à la lumière. Un moment qui laisse ses marques n'est pas nul et non avenu, et il faut donc tenter de voir comment il s'enchaîne en tradition, surtout quand son emplacement géographique l'approche de notre point d'observation. C'est pourquoi nous avons demandé la photographie du «Blaise Pascal au milieu de ses contradicteurs» de Jean-Louis Charbonnel (1878), qui dormait dans les réserves du Musée de Riom (fig. 2).

Albert Ojardias s'est amusé des "copies et copies de copies» foisonnant «dans la province de Pascal », à partir de la peinture de Quesnel réputée la plus proche du décès de Pascal, mais il parait tenté de prendre au sérieux «quelques autres portraits de garantie moindre que les authentiques, mais très supérieurs au genre capricieux de l'approximation ${ }^{2}$ ». Ces moindres " garanties " s'attachaient au début du XX $\mathrm{XX}^{\mathrm{e}}$ siècle à des objets qui étaient encore à la portée des yeux : leurs pistes - nous en avons fait plusieurs expériences - paraissent maintenant complètement effacées... Cependant, comme il ne faut jamais désespérer, nous appelons nos associés, amis et correspondants, à traquer les souvenirs familiaux et à alerter le CIBP toutes les fois qu'un objet à propos duquel on a une fois ou l'autre associé l'existence de Pascal ou des siens, leur est signalé. L'enchaînement peut se révéler imaginaire, ou bien il ne pourra pas être renoué, l'objet ne se retrouvant pas du tout ou bien les présomptions d'authenticité n'étant pas confirmées, mais l'on découvre toujours quelque chose de significatif, et l'effort de remonter le temps, pas après pas, a cette vertu de nous accoutumer à chercher à regarder le monde comme Pascal a pu le voir.

9 Nous voilà parvenus à la couche ancienne. À Gilberte et Jacqueline Pascal, les Amis de Port-Royal et le CIBP ont consacré un colloque à l'automne 1981, dont les actes ${ }^{3}$ reproduisent un portrait de chacune d'elles. Celui de Gilberte, qui est fixé à ClermontFerrand ${ }^{4}$, est très révélateur et son authenticité n'a jamais soulevé de doute; celui de Jacqueline au musée des ruines de Port-Royal est dans le cas des "moindres garanties ", ce qui ne veut pas dire qu'il ne comporte aucune vérité, plastique ou d'ordre spirituel. Albert Ojardias n'en a pas connu d'autres. M. Bernard Dorival dans sa grande étude sur Philippe de Champaigne signa le parmi les « Euvres inauthentiques et douteuses un autre portrait de Jacqueline dit « très bonne peinture de maitre » par le catalogue du vendeur ${ }^{5}$.

Pour Marguerite Périer la situation est bien meilleure. L'ex-voto où elle figure à genoux devant le reliquaire de la Sainte-Épine est connu de tout le monde. Si nous le présentons quand même, accompagné d'un document de comparaison, (fig. 3 et fig. 4), c'est qu'elle est notre héroïne de ce jour. Puisque nous la reconnaissons comme la principale donatrice du CIBP elle doit figurer en belle place dans notre album. Enfin nous avons le plaisir de l'entourer de visages qui ont dû lui être familiers : deux documents inédits vont ramener parmi nous les hommes de sa famille (fig. 5, 5 bis et 6 ). 


\section{Illustrations Interprétations concernant Pascal}

11 Il arrive plus souvent que les peintres prennent la plume pour expliquer les principes de leur art, que les écrivains ne prennent le crayon ou le pinceau afin de traduire sensiblement leurs conceptions ou leurs rêves. Mais les deux cas expriment également bien l'admirable connexion intérieure de la créature du sixième jour. Ut pictura, poesis : en quelque sorte qu'on tourne l'adage, on trouve un point d'attache commun dans l'esprit: la peinture est « chose mentale » disait Léonard de Vinci, et le style, osons-nous ajouter, est un plaisir de la main. Deux fois heureux celui chez qui la correspondance entre l'instrument de la pensée et celui de la fabrication fournit deux pistes de réalisation, homo sapiens et homo faber résumés dans une vocation créatrice.

On dit « portrait » en littérature comme en peinture, et la ressemblance que poursuivent et l'écrivain et le peintre, ne se divise pas selon le physique et selon le moral, car même si les moralistes mondains du XVII ${ }^{e}$ siècle répartissaient les caractères en deux groupes, l'ordre fuit toujours et l'unité de la personne résiste à l'analyse. C'est par un seul et même trait que le portraitiste fait voir l'âme et les formes.

Fig. 1. Jean GUITTON. Portrait de Blaise Pascal.

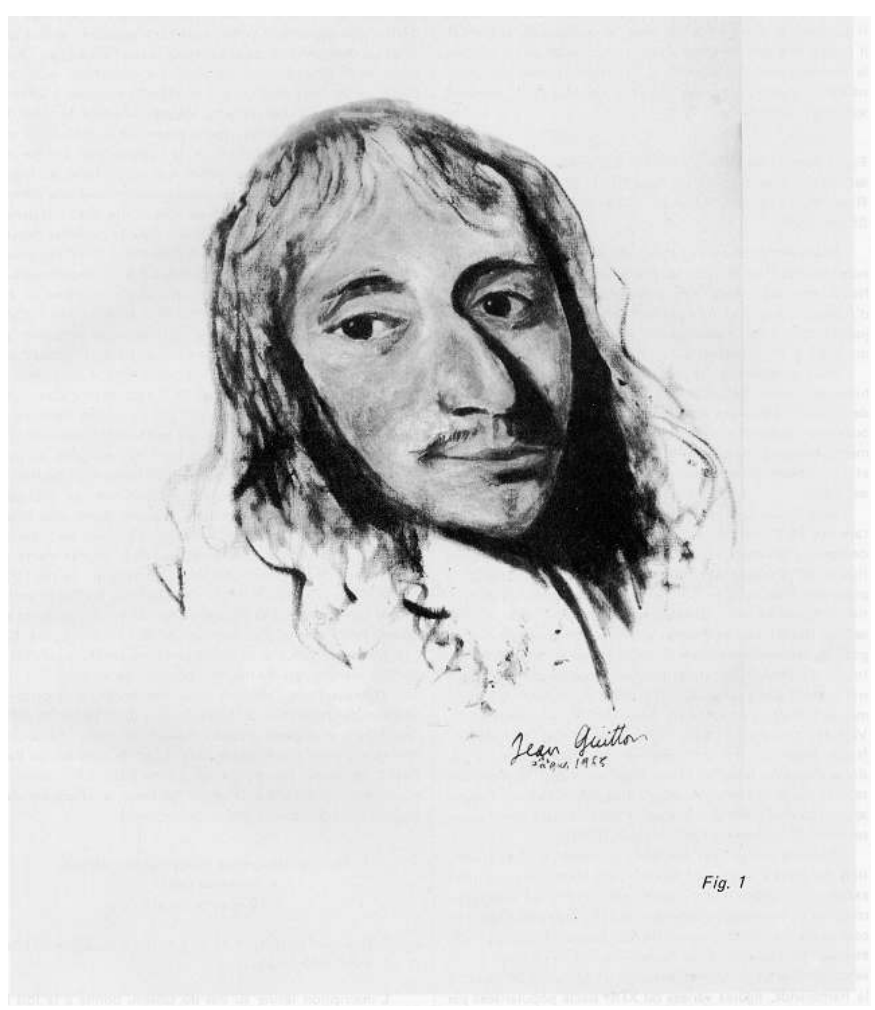

Huile sur toile. H. $62 \mathrm{~cm} \times 1.52$ cm, signée et datée : Pâques 1963. Photographie donnée par l'auteur. Traitée par Studio 23, 23 bd du Montparnasse, 75006 Paris.

«Je crois, dit M. Guitton, que si j'avais été peintre, je me serais exercé aux portraits. » Quand nous sommes entrée dans le bureau, ou l'atelier, de M. Guitton, nous avons compris pourquoi tant de ses œuvres s'appellent «portrait» composant un volume 
Portraits (1966) de ses Euvres complètes. Nous nous souvenons avec reconnaissance de ce Portrait de M. Pouget (1954), Grand prix de littérature, qui ouvrit à notre recherche encore jeune les profondeurs de la simplicité en exégèse, et nous trouvons notre joie en ce Portrait de Marthe Robin (1985) délicat et prophétique. Jean Guitton a fait avancer Pascal en " profil parallèle » avec Leibniz (1970), et Renan en comparaison avec Newmann. Chez lui pas de conflit entre la curiosité biographique et l'intellection pure. Ce qui importe, dit-il, c'est «la présence cachée d'une philosophie dans les portraits, et celle d'une pensée humaine dans les abstractions. Ou encore c'est la présence de la recherche critique à l'intérieur de l'acte de foi. » (Portraits, Préface, p. 8).

Jean Guitton a beaucoup vécu avec Pascal. L'idée d'évoquer sur la toile ce visage sur lequel on a si peu de documents certains, lui est venue dans une période heureuse de sa carrière. Élu à l'Académie française en 1961, il avait été délégué pour représenter cette compagnie aux fêtes du tricentenaire de la mort de Pascal qui furent célébrées de manière grandiose à Clermont-Ferrand en 1962. D'ailleurs Jean Guitton né le 18 août 1901 à Saint-Étienne, tient à reconnaître ses attaches auvergnates. Son arrière-grand-père maternel, Adolphe Ancelot, (1815-1889) substitut du procureur général puis avocat général à Riom, était membre de l'Académie des Sciences, Belles Lettres et Arts de Clermont-Ferrand. La France du Centre attache Jean Guitton de tous les côtés. Il a été professeur à Moulins. Il est souvent venu à Clermont-Ferrand, pour le pire (mobilisé et puis fait prisonnier en ces lieux) comme pour le meilleur. Et d'ailleurs un lien philosophique s'entrelace au fil de ses souvenirs. Son maître Henri Bergson a été professeur à Clermont de 1885 à 1890 et Guitton consacre un chapitre de sa Vocation de Bergson (1960) à "ces années d'apprentissage en Auvergne » qui furent décisives et qui furent heureuses.

C'est à Nice, rue Fragonard, pendant les vacances de Pâques 1963 que Jean Guitton décida de faire un portrait de Pascal, présenté en esquisse sans le corps, à l'instar du célèbre Bonaparte de David. Il a employé une huile très diluée et des teintes d'ocre jaune qu'il voulait automnales, sur la toile laissée en fond blanc. La pose lui a été fournie par un portrait attribué à Philippe de Champaigne où le possesseur, Ulysse Moussali, voyait Le Vrai Visage de Blaise Pascal (1952). Ce visage a orné la couverture d'une édition de poche des Pensées. Elle figure toujours sur celle de Pascal par lui-même, d'Albert Béguin, et la toile elle-même a été acquise pour le Musée national des Granges de Port-Royal. On juge le plus souvent que l'origine de cette peinture ne permet pas l'identification à Pascal, et qu'il faut plutôt reconnaître Isaac-Louis Le Maître de Sacy ${ }^{6}$. Mais en tout cas il s'agit d'un ami de PortRoyal. Que l'auteur soit Philippe de Champaigne ou non, ce regard doux et interrogatif ne se laisse pas oublier. Pour la morphologie du visage, Jean Guitton s'est fondé sur le contremoulage du masque mortuaire que chacun connaît et que nous affrontions dans la livraison $\mathrm{n}^{\circ} 6$ de ce Courrier.

Je regarde cette esquisse, si longtemps portée en méditation, et s'il m'est permis de donner ma réponse à l'irruption de cette physionomie, je dirais que j'y trouve une conviction de solitude qui provoque chacun à l'interroger comme sa propre conscience.

M. Jean Guitton est de ceux qui ont accueilli avec joie la création de notre centre d'information et de documentation. Il est membre bienfaiteur de notre association de soutien et il nous a donné la présente photographie de sa peinture. Mais le bienfait pour lequel nous lui portons le plus de reconnaissance c'est la généreuse franchise par laquelle il transmet son amour pour Pascal. 
Fig. 2. Jean-Louis CHARBONNEL, Blaise Pascal au milieu de ses contradicteurs.

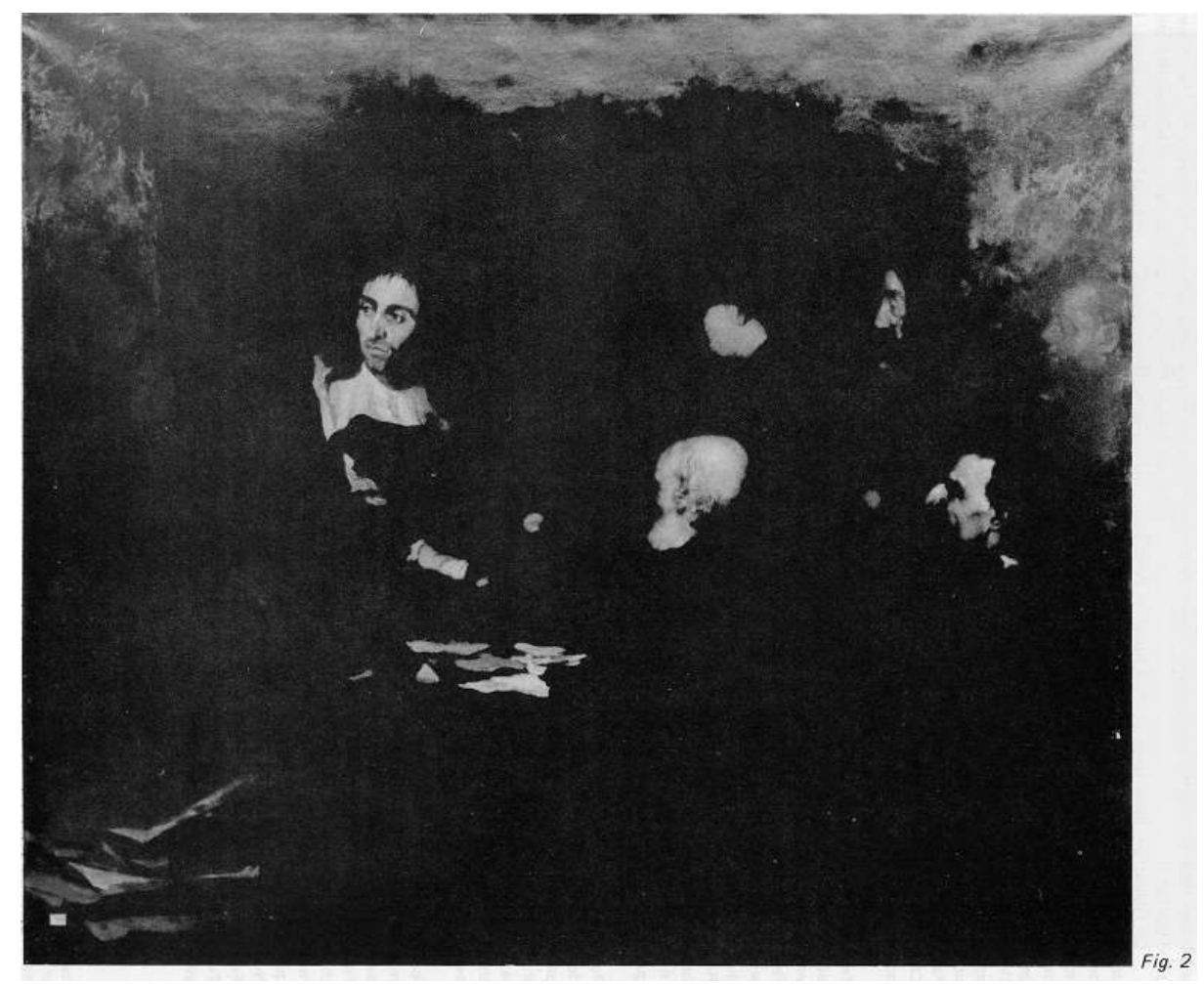

Huile sur toile. H. 1,98 m x L. 2,29 m. Riom, Musée Mandet, Réserves, n D 878. 1. 3.

Cliché Franck Studio, Riom.

Nous avons connu l'existence de cette peinture par une note de Jean Guitton dans ses Profils parallèles, 1970, p. 209. Notre ami, ayant beaucoup fréquenté le territoire pascalien d'Auvergne, s'est fait très précisément notre collaborateur. Il jugeait cette toile «peu connue » comme «digne d'être remarquée. » Nos lecteurs se rangeront d'emblée à son avis.

Pour comprendre les intentions du peintre, une notice historique serait fort utile. Nous n'avons trouvé que fort peu de choses ${ }^{7}$, mais la présente publication faisant office de lettre ouverte et de questionnement, nous espérons que des renseignements surviendront qui éclaireront la biographie, la carrière et l'esthétique de ce peintre oublié et qui de surcroît était auvergnat.

Jean-Louis Charbonnel est né à Bélinais-Paulhac (Cantal); il a été l'élève de Léon Cogniet, de Jean-Léon Gérôme et de Carolus Duran. Il a exposé au Salon de 1868 à 1882, " des figures et des portraits " (Bénézit). Au Salon de 1878 il présentait donc, à la section peinture, cette composition relative à Pascal ( $\left.\mathrm{n}^{\circ} 464\right)$, un Meâ culpâ, étude ( $\left.{ }^{\circ} 465\right)$, et à la section des dessins un Portrait de Mgr Lamouroux de Pompignac, évêque de Saint-Flour (n - 2618) et un Portrait de Louise ( $n^{\circ}$ 2619). Bénézit lui attribue : Apothéose de sainte Marguerite (1868) ; Deux Grigous (1872) ; Portrait de l'artiste par lui-même (1872) ; La Sortie du bain (1873); Aspasie (1874); Vice et Innocence (1880); Portrait de M. Le Saint (1882). Nous avons vu les photographies des tableaux suivants: Blaise Pascal au milieu de ses contradicteurs, Les Deux grigous, tableau qui doit être au musée de Bagnières; Aspasie, Femme se penchant (1878) et une superbe tête d'artiste romantique, sans intitulé, dédiée « à l'ami Pompon, 1881 ». 
de ces œuvres qui peut le mieux éclairer l'intention du groupe entourant Pascal, c'est plutôt - qu'on nous excuse - la composition d'Aspasie, par son effet de surprise recherchée et son anachronisme provocant: une femme nue - la courtisane d'Athènes au centre optique du tableau, de profil étendant le bras droit au milieu d'un cercle de gens vêtus, très vêtus, de tous les milieux et de toutes les époques: bourgeois à la Rembrandt, figures variées du XIX siècle popularisées par la peinture. On évoque forcément Le Déjeuner sur l'herbe de Manet, qui remontait à 1863, mais l'effet scandaleux recherché paraît ici plus intellectuel. Si Aspasie n'appartient à aucun temps, c'est probablement pour signifier qu'elle est éternelle.

Dans notre tableau, au rebours, la figure centrale porte la marque de son temps : Pascal est bien vêtu comme on peut supposer qu'il l'était. Les autres personnages portent des costumes plus vaguement datés, tous ecclésiastiques autant que l'état de détérioration de la peinture permet d'en juger : manteaux enveloppants, frocs de moines à capuchon, deux chapeaux cardinalices mais qui ont oublié d'être rouges. L'effet de lumière traversant obliquement, frappant le visage, le collet, les manchettes de Pascal, les papiers placés sur la table et la tête blanche d'un moine, apparente la composition à celle des Pèlerins d'Emmaüs de Rembrandt, version de 1648, au Musée du Louvre. Mais Charbonnel a voulu le déséquilibre: six personnages à la gauche de Pascal, deux à sa droite. Cela fait penser au mot du disciple de M. Le Moine dans la première Provinciale: «Nous sommes le plus grand nombre, et s'il est besoin nous ferons venir tant de cordeliers que nous l'emporterons ", et à l'affirmation de l'épistolier dans la dix-septième : "J'ai dit que je suis seul.» Comme Pascal a écrit les Provinciales sous l'anonymat, il n'a jamais rencontré en personne ses contradicteurs et le peintre n'a donc pas voulu illustrer une anecdote historique. Mais que fait donc Pascal au milieu de tous ces doctes ecclésiastiques? M. Descotes voit dans cette réunion un souvenir de Jésus enfant parmi les docteurs du Temple. Les savants théologiens qui entourent Pascal sont tous plus âgés que lui. Ils se tiennent dans des attitudes où peut entrer de la réserve mais sans la moindre marque d'hostilité. Ils semblent attendre de lui la parole lumineuse qui soulagera leurs tensions, hommes bien réels au point que chaque figure pourrait être un portrait. Or Pascal n'est pas en train de répondre. Il serre même les lèvres. Ses deux solides mains, de vraies mains de sculpteur, étreignant un livre qui ne peut être que la Bible, il respire la lutte, mais une lutte intérieure plutôt qu'une controverse. Cet ardent visage de moine espagnol est tourné vers l'écoute. Oui écoute-t-il ? Est-ce le discours d'un sage qui serait placé à sa droite, hors du cadre, ou plutôt le docteur invisible qui n'a besoin d'occuper aucun lieu ?

Volontiers nous verrions ici la mise en scène de cette injonction des Pensées : «Écoutez Dieu. » (Laf. 131 - Br. 434). Pascal laïc enseignant l'Église enseignante, telle me semble être la signification de cette toile. L'artiste, réaliste au confluent de toutes les écoles de peinture du XIXe siècle, a transcendé l'histoire par la vérité poétique, et d'une certaine façon il l'illumine par sa généreuse sympathie.

\section{Documents anciens anonymes concernant la famille Périer : Marguerite enfant}


Fig. 3. Marguerite enfant

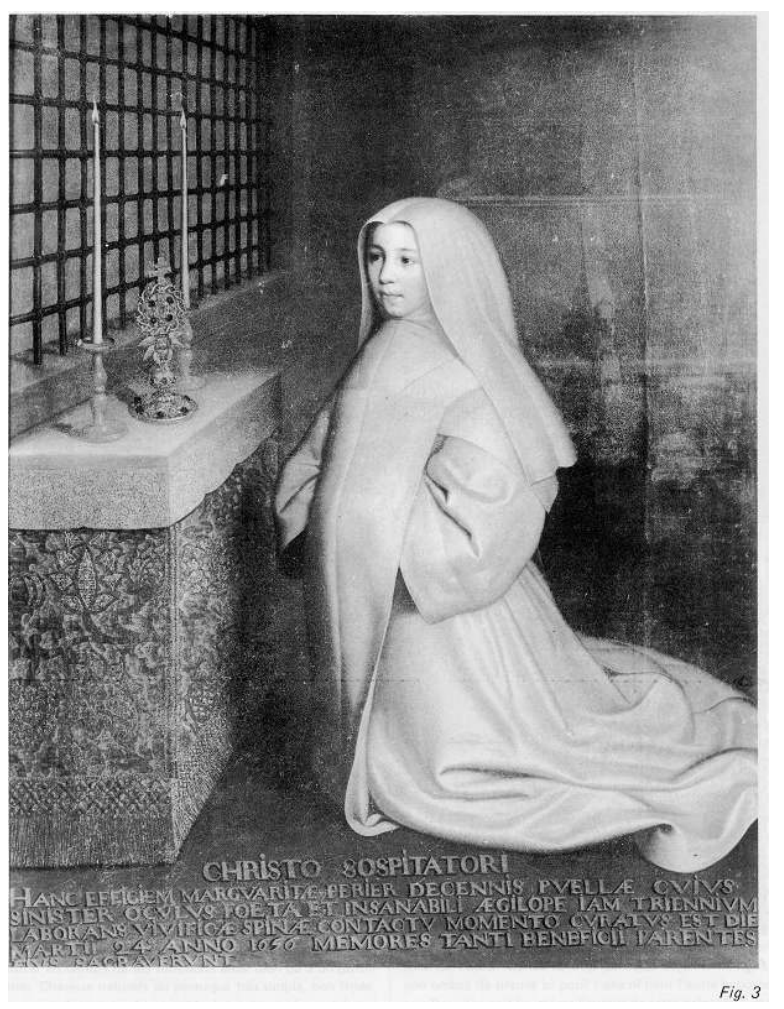

Huile sur toile. H. 1,42 m x L. 1,10 m. Église de Linas (Yvelines).

Cliché Giraudon. Claude Baudran, également miraculée de la Sainte Épine (en 1657), et une copie du tableau de Philippe de Champaigne Les deux religieuses, a été légué à la paroisse de Linas en 1842, par M. de Carnet La Bonnardière, gendre de M. Laideguive, janséniste notoire du XVIII ${ }^{e}$ siècle (Voir André HALLAYS, Le Pèlerinage de Port-Royal, 1909, pp. 293-308). Il a figuré dans de nombreuses expositions, notamment dans celle de la Bibliothèque nationale, Blaise Pascal, 1962, n 395 du catalogue. L'attribution à Philippe de Champaigne est maintenant exclue, mais on n'a avancé aucun nom pour le peintre à qui Florin Périer aura passé la commande.

le fait de ce tableau nous comprenons combien la famille Périer avait investi de son être dans cet événement reçu par elle comme miraculeux : la reconnaissance à garder est devenue comme la raison de vivre pour l'objet de cette célèbre faveur. La vieille demoiselle survivant à la gloire familiale ne sera donnée à la postérité qu'en petite fille, revêtue, pour la dignité de son action de grâce, de l'habit des novices de Port-Royal, alors qu'elle n'y a jamais été que pensionnaire. Quelle fut alors la divulgation de l'image? Les gravures, faciles à multiplier, étant laissées hors du compte, essayons de compter quelques exemplaires de la peinture qui ont été signalés. 
Périer, ayant traversé sa longue existence, saisissait-elle cette image d'elle-même par laquelle aurait dû éclater aux yeux de tous une certaine prédestination placée en sa famille? Elle qui avait suspendu à son chevet l'image du diacre Pâris ${ }^{11}$, mettait sans doute la bénéficiaire d'un miracle sans comparaison au-dessous du digne intercesseur, agent de miracles. Son testament de 1720 spécifie la destination des peintures religieuses lui appartenant et d'un tableau de reliques, mais il ne parle pas de peintures sur le sujet de son miracle. L'ex-voto réduit de taille devenait donc, comme elle dit, son «portrait habillé en petit habit de religieuse », une transmission du moi : il s'ensevelissait donc dans l'intimité d'un legs à sa femme de chambre ${ }^{12}$. Le testament d'ailleurs ne parle d'aucun portrait de sa famille. L'on sait pourtant que les hospices eurent - puisqu'ils l'ont encore - le portrait de $\mathrm{M}^{\mathrm{me}}$ Périer. M. Mesnard pense qu'un Florin Périer lui faisait probablement pendant ${ }^{13}$. Pourquoi n'y aurait-il pas eu d'autres portraits encore? La modestie, de consigne familiale, qui empêche d'en faire mention, ne seraitelle pas la composante d'une sorte de fierté (de race) construite à l'inverse de la commune vanité ? Dans ce milieu austère une absence de témoignage sur ce qui touche le moi personnel ne 
signifie pas que la personnalité propre n'a pas produit en son temps des marques effectives de son passage.

Fig. 4. Attribué à Philippe de Champaigne. Petite fille vêtue de bleu aux mains jointes.

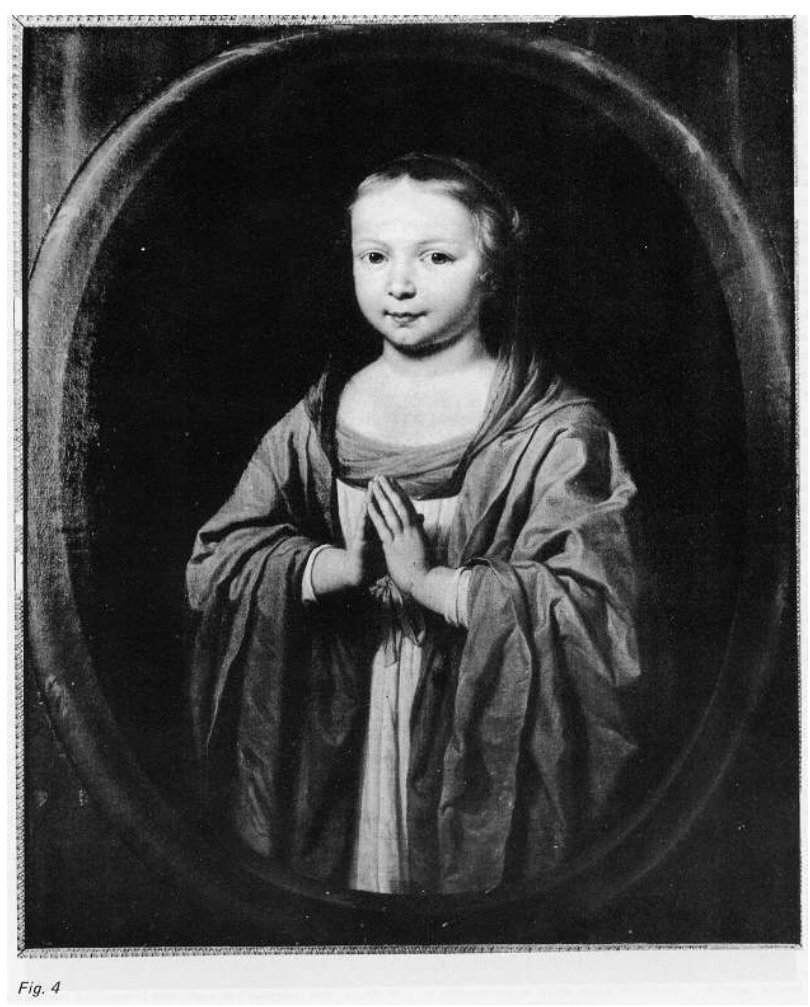

Huile sur toile, H. 0,70 m x L. 0,58 m. Musée du Louvre. Inventaire 1149.

Cliché Réunion des Musées nationaux.

C'est le Magasin pittoresque de $1848, \mathrm{t}$. XVI, qui a le premier affirmé que cette petite fille était Marguerite Périer ${ }^{14}$. La peinture d'excellente qualité peut bien sortir de l'atelier de Philippe de Champaigne et elle paraît être à peu près du même temps que le tableau de Linas. Il y a une certaine ressemblance des traits pour la forme du nez, la lèvre supérieure, le menton, mais cela ne serait-il pas dû à des habitudes de métier contemporaines? Cette charmante petite fille aux yeux bleus et aux cheveux blonds cendrés, éclatante de santé, paraît avoir moins de dix ans. Son costume, bleu et gris, n'appartient à la mode d'aucun temps : c'est un drapé et voile pour effigie de sainte. Car l'intention hagiographique la concerne elle-même et ne tend pas, comme dans le tableau de Linas, à mettre en valeur un acte du moment. Cette enfant incarne un modèle de prière. La peinture opère sur elle comme une affectueuse canonisation. S'agirait-il d'évoquer une enfant regrettée? Il semble en effet que la vieillesse ne doit jamais l'atteindre.

$\mathrm{M}^{\mathrm{me}}$ Thérèse Picquenard, conservateur du Musée national des Granges de Port-Royal, qui a bien voulu venir voir cette peinture avec moi dans les réserves du Louvre, pense qu'il ne s'agit pas de Marguerite Périer. C'est dommage. 
Fig. 5. Auteur inconnu. Portrait d'homme.

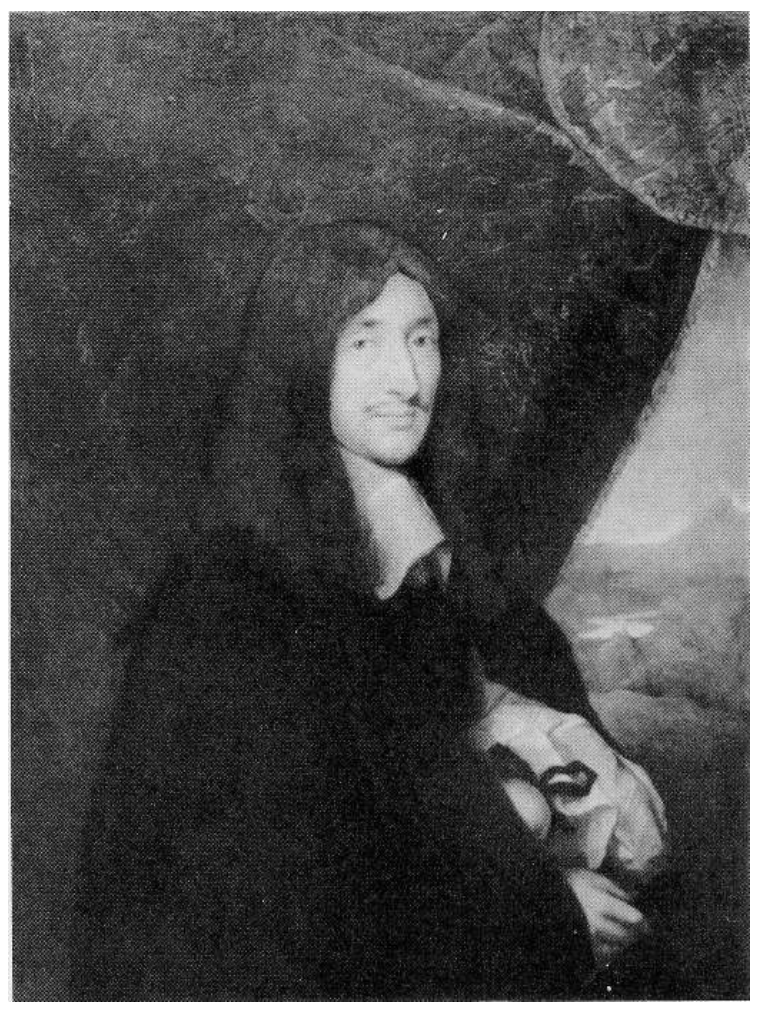

Peinture sur toile. H. 1,01 m x 1. 0,78 m. Collection particulière. Cliché Yves Morvan. 
Fig. 5 bis Auteur inconnu. Portrait d'homme.

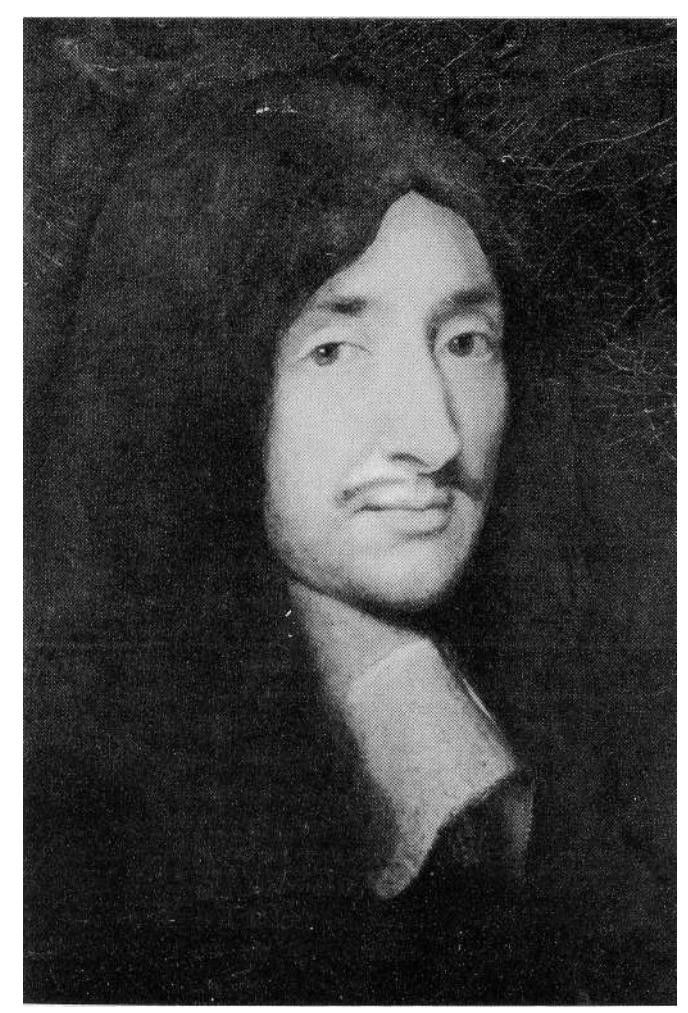

Peinture sur toile. H. 1,01 m x 1. 0,78 m. Collection particulière.

Cliché Yves Morvan.

La raison que nous avons eue en premier lieu de présenter cette toile c'est qu'elle nous a été annoncée comme un portrait de Florin Périer. Malheureusement la personne auteur de cette assertion a disparu de nos relations. Nous espérons que notre publicité de proche en proche la rejoindra et qu'elle nous éclairera sur les possibilités de cette identification.

Nos autres raisons, c'est que ce tableau a toujours été dans une ancienne famille d'Auvergne d'où un fervent amateur, alerté par la qualité du cadre, l'a recueilli en fort mauvais état. Enfin la raison qui pourrait suffire c'est la beauté, apparue après un simple nettoyage, de cette physionomie où se trouve représenté un moment de l'histoire où Blaise Pascal, beau-frère de Florin Périer, était en sa pleine maturité.

Le problème de la datation est donc le premier qui se pose et peut-être le seul que nous ayons quelques moyens de poser puisque le tableau lui-même en fournit les données. Le costume sombre, très sobre, illuminé par le rabat en toile couverte de dentelle avec un pompon visible, et par le bouffant de la chemise serrée au poignet par un ruban noir, peut convenir à un magistrat en dehors de ses fonctions aussi bien qu'à un gentilhomme. Cheveux naturels ou perruque très simple, non frisée, on ne peut distinguer. L'ensemble fut à la mode antérieurement au règne personnel de Louis XIV. Mais le détail le plus particulier nous a paru être la moustache fine et courte, s'épaississant vers le bas et ombrant la commissure des lèvres. Le faire du peintre sert également de repère. Le drapé $\mathrm{du}$ fond, le paysage lointain d'eau et de montagnes qui peut représenter une Italie de convention aussi bien que l'Auvergne, n'ont rien de caractéristique. Mais l'ovale fin du visage, la moustache typique, la tenue des mains et un je ne sais quoi de sensible, nous ont rappelé un souvenir : celui d'un présumé Nicolas Fouquet du Musée de Versailles (MV 
4259. Inv. 9220. H. $(1,01)$, L. $(0,80)$. Les portraits d'homme par Sébastien Bourdon dont nous avons examiné les photographies présentent ces mêmes caractéristiques. Citons «L'Homme aux rubans noirs» du Musée Fabre à Montpellier, l'Autoportrait du Musée Calvet à Avignon, un portrait de gentilhomme passé en vente à la Galerie Charpentier, le 18 janvier 1957, et ce portrait d'homme à Chicago, au musée The Art Institute, qui fut présenté à Paris en 1982, dans l'exposition La peinture française du XVII ${ }^{e}$ siècle dans les collections américaines (catalogue Réunion des Musées nationaux, planche p.191, notice p. 229) et qu'on suppose peint à Montpellier vers 1657. Sébastien Bourdon (Montpellier, février 1616 - Paris, 8 mai 1671) a séjourné en Suède dans les années 1652-1653. Il est le peintre de la reine Christine, la bienfaitrice de Pascal. Certes ce trop joli rapprochement ne fait pas une ombre de preuve ni pour l'une ni pour l'autre hypothèse : que Bourdon soit lui-même l'auteur de cette peinture - mais il est certain que l'artiste a voulu peindre comme Sébastien Bourdon - et que le sujet en soit Florin Périer. Qu'on ne s'imagine pas si élégant le gendre choisi par Étienne Pascal n'est pas une objection, mais sa date de naissance (1605) en est une bien forte... Quoi qu'il en soit on peut retenir que le goût pour ce style d'humanité était présent en Auvergne entre les années 1650 et 1660.

Et pour commenter cette séduisante image (qui nous a trop séduite ?) nous ne pouvons rien trouver de mieux que ce que le catalogue plus haut cité dit (p. 186) de Sébastien Bourdon lui-même. "Il aime multiplier et comme " casser » les plis des costumes de ses modèles, allonger à l'extrême leurs mains, donner à leur visage une expression réservée, distante et tourmentée. La gamme des couleurs noir, gris, blanc et parfois bleu, accentue l'austérité des portraits de cet artiste encore aujourd'hui si mal étudié. »

Fig. 6. Auteur inconnu. Étienne Périer. Médaillon de plâtre

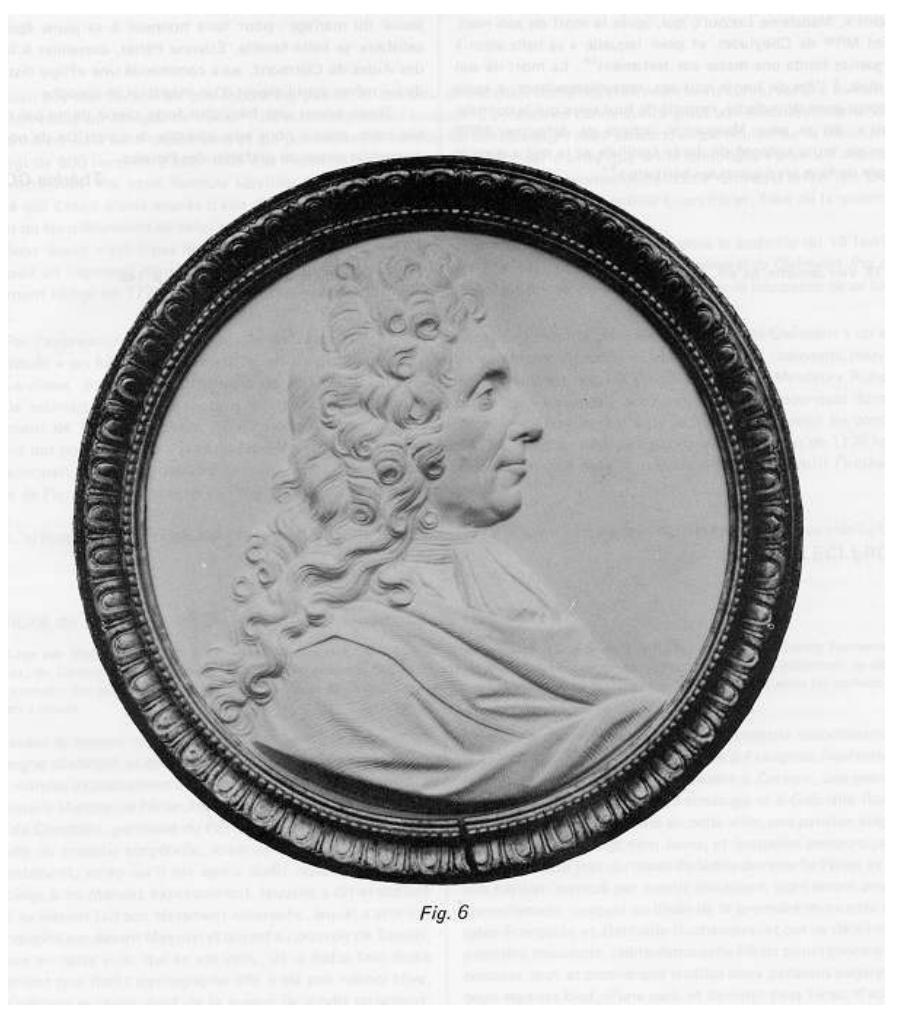

Diamètre 0,48 m, plus cadre 0,05 m.Collection particulière.

Cliché Studio Gibert, à Thiers. 
Cette fois nous osons donner un nom au modèle, parce que nous connaissons la transmission de l'objet. Il appartient à des arrière-neveux de Florin Périer, issus en droite ligne de sa sœur Marie Périer, épouse d'Antoine Aubert, receveur des tailles à Issoire, qui nous ont généreusement donné l'autorisation de le reproduire. Une petite fille de Marie Périer. Marie Aubert de Parpasset épousa Jean Morel de la Colombe, seigneur de la Chapelle (Chapelle-sur-Usson) par contrat passé dans le château de ses cousins, c'est-àdire à Bien-Assis, le 12 janvier 1700. Dans son testament Marguerite Périer se justifie de ne point faire Messieurs Aubert ses héritiers : « ce n'est point par animosité ni aversion », mais la volonté de sa mère était de faire des pauvres ses héritiers. Marguerite et son frère le chanoine eurent même scrupule «d'avoir dissipé tant de biens pour la famille de $\mathrm{M}^{\mathrm{rs}}$ Aubert ». C'était 12000 livres au contrat de mariage de Jean Aubert, payables après le décès des donataires, et 4000 livres pour sa fille $\mathrm{M}^{\mathrm{me}}$ de La Chapelle, dont le règlement avait déjà présenté bien des difficultés dès l'année du testament (1720) ${ }^{15}$. Après la mort de Marguerite, en 1733, dans les contestations qui suivirent entre les hospices de Clermont d'une part et d'autre part les mandataires et les parents de la défunte, il fut reconnu que les Aubert étaient les plus proches parents ${ }^{16}$. C'est donc tout naturellement que ce médaillon dut leur revenir parmi les objets sans appréciation vénale. La tradition chez les descendants veut qu'il provienne de Bien-Assis. En tout cas son déplacement n'offrait aucune difficulté, car le cadre, fort beau, fait d'une seule pièce (le chauffage central l'a fait fendre), ne porte aucune trace d'insertion dans une boiserie.

Ce médaillon représente donc, de profil, un homme de la famille Périer. Lequel? Le costume, manteau drapé, cravate nouée lâchement, étant stylisé n'apporte pas d'indice bien précis de datation; la perruque, majestueuse sans être énorme, soigneusement bouclée, a pu convenir à des magistrats de plusieurs générations. Elle correspond en tout cas, nous a dit $\mathrm{M}^{\text {elle }}$ Delpierre, conservateur honoraire au Musée du Costume et de la Mode (Palais Galliéra) aux années 1670 à 1680 où le fils aîné de Florin Périer exerçait, depuis 1669, la fonction de Conseiller à la Cour des Aides de Clermont. La présentation en profil et le style noble diminuent l'empreinte de l'âge, mais il s'agit certainement d'un homme jeune ou dans la force de l'âge, et certainement en dessous de cinquante ans. Florin Périer, mort en 1672, à l'âge de 67 ans, ne peut donc avoir été le modèle. Comme on n'a aucun témoignage sur le physique de son fils Étienne, on peut accepter qu'il ait demandé à l'artiste de faire ressemblant. Le ferme modelé des traits, le pli du sourire, la verrue sur la joue droite, tendent en effet à faire croire au réalisme. L'expression est celle d'une intelligence ferme, un peu portée à la moquerie. Était-ce celle du modèle, ou la tendance de l'artiste vers l'esprit nouveau?

41 Étienne Périer, baptisé le 15 avril 1642 à Rouen, paroisse Saint-Godard, décédé à Clermont, le 11 mai 1680, sur la paroisse Saint-Genès, fut certainement le plus intellectuel des cinq enfants Périer ${ }^{17}$ Son oncle Pascal le garda un certain temps auprès de lui et s'occupa de son éducation. Étienne fit ensuite des études de mathématiques et de droit. Quand il s'agit d'éditer l'apologie inachevée, $\mathrm{M}^{\mathrm{me}}$ Périer écarta la préface préparée par Port-Royal et lui en substitua une autre rédigée par son fils aîné (âgé de 28 ans) qui, pour ne pas faire de peine aux amis, déclara l'avoir reçue de Clermont « toute faite ».

42 C'est ce que sa mère appelle une "finesse ». Étienne correspondit avec Leibniz qui l'encourageait à publier les papiers scientifiques laissés par son oncle. Ses deux frères ayant embrassé l'état ecclésiastique, eut-il, en tant qu'aîné de la famille, des ambitions mondaines? Ce fut le seul qui envisagea de se marier et après avoir refusé un très riche parti, il épousa, en 1678, "une demoiselle de condition qui avait beaucoup d'esprit ", 
Madeleine Lecourt, qui, après la mort de son mari, devint $\mathrm{M}^{\mathrm{me}}$ de Cheyladet, et pour laquelle « sa belle-sœur » Marguerite fonda une messe par testament ${ }^{18}$. La mort de son fils aîné, à l'âge de trente-huit ans, sans descendance, «après quatorze jours de maladie, regretté de tous ceux qui le connaissaient », dit sa sœur Marguerite, acheva de détourner $\mathrm{M}^{\text {me }}$ Périer de toute volonté de durée familiale et la mit «dans le dessein de faire les pauvres ses héritiers ${ }^{19} »$.

43 Alors que signifie ce coûteux médaillon, travail d'art rarement commandé dans les familles bourgeoises? On ne peut même pas dire: Sic transit gloria mundi, tellement dans cette courte vie la gloire se montre peu, alors qu'un tel portrait connote un homme imposant. Y eut-il l'occasion du passage d'un artiste habile et encore peu coté ? L'hypothèse qui, à notre avis, se soutient le mieux, serait l'établissement social du mariage: pour faire honneur à sa jeune épouse et satisfaire sa belle-famille, Étienne Périer, conseiller à la Cour des Aides de Clermont, aura commandé une effigie distinguée de lui-même sous l'aspect d'un magistrat philosophe.

Nous avions une bien plus forte raison de ne pas oublier son nom, mais il nous sera agréable de connaître de nos yeux un certain visage du préfacier des Pensées.

\section{NOTES}

1. Albert OJARDIAS, « Divers portraits de Pascal et des siens ", Gazette des Beaux-Arts, 1910, t. 11, p. 195-207, et tiré à part (p. 10).

2. Ojardias, op. cit., p. 10 et 12.

3. Deux grandes figures d'Auvergne. Gilberte et Jacqueline Pascal. Chroniques de Port- Royal, $\mathrm{n}^{\circ} 31$, et Amis du CIBP. Paris et Clermont-Ferrand, couverture et p. 47. Portrait de Jacqueline, p. 103. Commentaires respectivement de Gérard Tisserand et de Thérèse Picquenard.

4. Au Musée du Ranquet. Appartient aux hospices de Clermont-Ferrand, légataires de Marguerite Périer.

5. Bernard DORIVAL. Philippe de Champaigne. 1976, t. 11, $\mathrm{n}^{\circ}$ 2014. Vente à Paris, 10-11 mai 1895, $\mathrm{n}^{\circ}$ 13. Sans les dimensions. Pas de reproduction.

6. Voir Bernard Dorival, op. cit., t. 11, $\mathrm{n}^{\circ} 214$. et «Recherches sur l'iconographie de Pascal : le prétendu portrait de Philippe de Champaigne ", La Revue des Arts, (oct.-déc. 1956), pp. 167-168 et 731 ; Ulysse MOUSSALI, Le Vrai visage de Blaise Pascal, 1952 ; ( L'iconographie de Blaise Pascal », La Revue des Arts, 1957, pp. 76-84; Jean MESNARD, Blaise Pascal. Euvres complètes, t. 1, 1964, ( L'iconographie pascalienne ». p. 411-414.

7. Nos sources : BÉNÉZIT (Emmanuel Charles), Dictionnaire critique et historique des peintres, Paris,

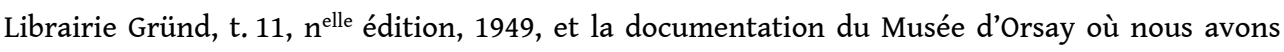
reçu le meilleur accueil. Consulté l'Album Michelez. Achats et commandes de l'État, et Explication des ouvrages de peinture, sculpture, architecture, gravure et lithographie des artistes vivants, exposés au Palais des Champs-Élysées le 25 mai 1878. Paris, 1 imprimerie nationale, 1878.

8. Élie JALOUSTRE, "Une nièce de Pascal, Marguerite Périer", dans Bulletin historique et scientifique de l'Auvergne, 1901, pp. 68 - 96 et 102- 147, à la p. 72. D'après le factum de l'avocat Quériau. Jaloustre donne en photographie, entre p. 68 et 69 , le buste d'une peinture qu'il assure datée de 1664. - Voir plus loin. 
9. Ambroise TARDIEU, Dictionnaire iconographique de l'ancienne Auvergne. 1904, p. 157.

10. Jean MESNARD, dans Pascal. Euvres complètes. t. 1. 1964. «L'iconographie pascalienne ». p. 415. - Une signature « Saigne pinxit 1714 » se trouve, cinquante ans après, au dos du portrait de Jean Poisson, chevalier seigneur de Lempdes, appartenant M. de Keating.

11. D'après Jaloustre, op.cit., p. 127 - 128 et 131. On peut voir le commentaire de Sainte-Beuve, Port-Royal, I. III, chap. XII (éd. de la Pléiade, t. II, p. 196-197.

12. Sur le legs à Hélène Bequet, voir Jaloustre, op. cit., p. 92 ; Ojardias, op. cit., p. 16-17 ; Mesnard, loc. cit. Hélène Becquet figure au testament publié par Jaloustre, p. 142-143. Jaloustre date le codicille du legs du 20 décembre 1720. Il n'indique malheureusement pas sa localisation, et les deux qu'il publie sont datés des 4 décembre 1720 et 23 mai 1721.

13. Mesnard, op. cit., p. 414.

14. Signalé par Jaloustre, op. cit., p. 72.

15. Dans Jaloustre, op. cit., p. 144, 145, 140, 145.

16. Ibid., p. 139.

17. Voir Blaise Pascal « auvergnat ». La famille à l'œuvre. Catalogue de l'exposition de Clermont, 1981 (au CIBP), p.73-76, et Marguerite Périer, Mémoire sur sa famille, dans Mesnard, op.cit. I, p. 1083-1084.

18. Voir Jaloustre, op. cit., p. 88 et 145.

19. Testament de Marguerite, Ibid., p. 145.

\section{AUTEUR}

\section{THÉRÈSE GOYET}

Professeur de l'Université II de Clermont-Ferrand 